\title{
Desigualdades y convergencias sociales en materia sexual y reproductiva entre adolescentes en Chile: Novedades de la Encuesta Nacional de Juventud 2009
}

\author{
JORGE RODRÍGUEZ ${ }^{(1)}$ y PAZ ROBLEDO ${ }^{(2)}$
}

\section{INTRODUCCIÓN}

La fecundidad adolescente, que operativamente corresponde a la que se produce entre los 15 y los 19 años ${ }^{a}$, es la que menos ha bajado en Chile en los últimos 45 años y, en la actualidad, es la que, por lejos, presenta el mayor nivel de desigualdad socioeconómica. En efecto, la fecundidad adolescente en 2009 era un $20 \%$ menor que la registrada en 1960 , mientras que la tasa global de fecundidad era un $60 \%$ menor. Más aún, como se mostrará en el texto, entre 2004 y 2009 la tasa de fecundidad del grupo de 15 a 19 años ha repuntado. Por su parte, la tasa global de fecundidad del grupo socioeconómico más pobre es, a lo más, el doble de la que registra el grupo más acomodado, pero esta relación es 10 a 1 en el caso de la maternidad adolescente ${ }^{1-5}$. Por otro lado, la fecundidad adolescente está asociada a un conjunto de adversidades específicas por riesgos de salud más elevados ${ }^{6}$, mayores probabilidades de deserción escolar y de peor desempeño escolar ${ }^{7,8}$, más probabilidades de ausencia de padre ${ }^{9}$, mayor frecuencia de embarazo por violencia o abuso ${ }^{10,11}$, menor madurez sicológica y habilidades sociales para enfrentar la crianza ${ }^{12,13}$ y por mayores dificultades para compatibilizar la maternidad con el trabajo remunerado ${ }^{14-18}$. La confluencia de estos factores: resistencia a la baja, desigualdad elevada y pertinaz, y adversidades biosicosociales, hacen de la maternidad adolescente una prioridad para las políticas públicas de salud, educación y juventud en Chile.

\section{MARCO CONCEPTUAL}

No existe un marco conceptual satisfactorio para explicar la resistencia al descenso de la fecundidad adolescente en América Latina y en Chile. En la región, han operado los principales componentes de la denominada modernización socioeconómica (urbanización, industrialización y terciarización de la economía, masificación de la educación, expansión de los medios de comunicación masivos), los que si bien han tenido debilidades y precariedades, en comparación con la experiencias de los países actualmente desarrollados, han -de todas formas-, contribuido a jalonar la transición demográfica y el descenso sostenido de la fecundidad total ${ }^{19-21}$. Dado que lo que ha sido válido para las mujeres jóvenes y adultas no lo ha sido para las adolescentes, la pregunta a responder es qué hace diferentes a las adolescentes del resto de las mujeres en materia reproductiva. Entre las líneas de respuesta, destacamos:

a. Estatus ciudadano, social y cultural: a diferencia de las/los jóvenes y adultas, las/los adolescentes tienen limitaciones legales y barreras sociales en materia de actividad sexual y de nupcialidad. Aunque en el caso de Chile la restricción legal no afectaría a la fecundidad del grupo de 15 a 19 años de edad (porque la imputación de violación o abuso refiere a la actividad sexual con personas menores de 14 años), las restricciones sociales sí lo harían, porque aún predomina la reprobación social

(1) Centro Latinoamericano y Caribeño de Demografía (Celade). División de Población de la Cepal. Avda Dag Hammarskjold 3477. Vitacura. Santiago. Chile. jorge.rodriguez@cepal.org

(2) Unidad de Adolescencia Clínica Alemana de Santiago y Centro de Salud Integral del Adolescente Rucahueche (Usach). Santiago. Chile.

(a) En este trabajo no se abordará la fecundidad precoz, que corresponde a la que ocurre antes de los 15 años. 
de la actividad sexual de los adolescentes y la negación de su condición de sujetos sexuales, lo que en la práctica se traduce en actividad sexual oculta, oportunista y con barreras para el acceso a métodos anticonceptivos

b. Control familiar: a diferencia de las/los jóvenes y adultas, las/los adolescentes tienen relaciones de dependencia legal, económica y sicosocial con sus padres. Cuando este control es efectivo (y se complementa con el control social y cultural antes comentado) y es restrictivo de la actividad sexual durante la adolescencia (típicamente porque sólo se acepta actividad sexual femenina intramarital y no se promueve el matrimonio temprano), las tasas de fecundidad adolescente suelen ser bajas. Pero cuando este control es restrictivo de la actividad sexual y aún así las y los adolescentes tienen actividad sexual premarital, el resultado puede ser fecundidad adolescente alta, no deseada (o no planificada) y no marital. Que este resultado se concrete dependerá, en gran medida, del papel del Estado y, eventualmente, de actores de la sociedad civil en materia de prevención del embarazo adolescente no planificado.

c. Definición identitaria: aunque para todas las personas tener hijos puede ser un aspecto central de su identidad adulta, en el caso de las/los adolescentes es un hito crucial que modifica radicalmente su condición social y que propicia una identidad específica, tarea central de la adolescencia como etapa. De esta manera, algunas adolescentes pueden considerar la maternidad como una opción para lograr simultáneamente una identidad personal y social específica (la de madre), en un contexto de falta de oportunidades de desarrollo personal y social.

d. Inmadurez, influencias exógenas y especificidades de género: a diferencia de las/los jóvenes y adulta las/los adolescentes están en pleno proceso de desarrollo biosicosocial y, por lo mismo, tienen poca experiencia en relaciones de pareja, conocimientos parciales y muchas veces míticos sobre la sexualidad y falta de manejo en materia de impulsos. Además, suelen tener sensación de omnipotencia e invulnerabilidad y les cuesta más planificar sus comportamientos. Por ello, les resulta más complejo decidir responsablemente en materia sexual y anticonceptiva. Por otra parte, suelen ser altamente influenciables por la opinión del grupo de pares y por los medios de comunicación masivos y las redes sociales que frecuentan, que en muchos casos transmiten mensajes marcadamente erotizados $\mathrm{y}$, a la vez, descuidados en materia de prevención, lo que incide en sus comportamientos sexuales y anticonceptivos. En materia de género, hay una asimetría valórica, por cuanto se estimula la actividad sexual masculina pero se estigmatiza la femenina. En esa misma línea, las mujeres suelen estar en condiciones de inferioridad para negociaciones relativas a la iniciación sexual y al uso de anticoncepción. Por ello, las adolescentes suelen experimentar más limitaciones que los hombres en materia sexual y anticonceptiva.

e. Desigualdad, pobreza y proyecto de vida: a diferencia de las/los jóvenes y adultas, las/los adolescentes están en plena definición de su futuro vital, de su proyecto de vida. Cuando las desigualdades sociales son elevadas, como ocurre en Chile, las oportunidades y proyectos de vida son muy limitados para los grupos de bajo nivel socioeconómico. Así, para estos grupos la maternidad adolescente no aparece como una interferencia grave para el logro de proyectos que están fuera de su alcance. Por ende, hay pocos incentivos para evitarla.

Estas diferencias entre las adolescentes y el resto de las mujeres se han presentado de manera esquemática y desvinculada sólo para efectos analíticos, ya que cada una es más compleja que lo expuesto y entre ellas hay nexos significativos. El propósito de esta pre- 
sentación gruesa es simplemente extraer algunas hipótesis asociadas a cada una de estas diferencias para evaluarlas empíricamente. En otros trabajos ${ }^{22}$ se ha avanzado en propuestas teóricas integrales para entender la fecundidad adolescente y su evolución en América Latina. Se ha usado el concepto de "modernidad sexual truncada" como principio explicativo principal y se ha complementado por factores como la desigualdad socioeconómica, las especificidades biosicosociales de la adolescencia y la acción de cuatro instituciones claves: familia, escuela, establecimiento sanitario y Estado (legislación, administración y políticas públicas). Este marco tiene un grado de abstracción elevado y contrastarlo empíricamente requiere de una amplia gama de fuentes de datos (algunas no disponibles) y de procedimientos multidisciplinarios aún no implementados.

Por ello, una forma alternativa de avanzar en el cotejo empírico que requiere todo marco conceptual es mediante respuestas a preguntas más acotadas que abonen a la discusión conceptual y a la especificación de las hipótesis más complejas. En esa línea, en este trabajo se aprovecharán los microdatos de la Enaju 2009 para responder preguntas que pueden aportar al debate sobre las especificidades de la reproducción durante la adolescencia. Estas preguntas atañen a: i) la persistencia de la desigualdad reproductiva durante la adolescencia; ii) la comparación entre las desigualdades socioeconómicas en materia de iniciación y actividad sexual, por un lado, y actividad sexual protegida, por otro, entre adolescentes; de este cotejo se concluirá cuál de las dos importa más para la desigualdad reproductiva en la adolescencia; iii) el sentido y el nivel de la desigualdad socioeconómica en el uso de recurrencia al aborto; iv) el sentido y el nivel de la desigualdad socioeconómica del embarazo no planificado.

\section{FUENTES DE DATOS Y METODOLOGÍA}

Las fuentes de información para el análisis de las variables intermedias de la fecundidad adolescente en Chile son escasas y limitadas.
En este trabajo se usa como fuente principal la sexta encuesta nacional de juventud (Enaju 2009) levantada por el Instituto Nacional de la Juventud (Injuv) en 2009, la que se complementa con otras encuestas de esta serie y estadísticas vitales.

La metodología es cuantitativa y se basa en el procesamiento de las bases de microdatos para obtener un conjunto de indicadores técnicamente pertinentes, a saber: i) porcentaje de personas de 15 a 19 años que tienen hijos; ii) porcentaje de personas de 15 a 19 años que han tenido relaciones sexuales penetrativas; iii) porcentaje de personas de 15 a 19 años que usaron anticonceptivos en su primera relación sexual (sobre el total de iniciadas sexualmente); iv) porcentaje de personas de 15 a 19 años que han tenido relaciones sexuales en los últimos 12 meses; v) porcentaje de personas de 15 a 19 años que usaron anticonceptivos en su última relación sexual (sobre el total de personas de esa edad iniciadas sexualmente); vi) porcentaje de personas de 15 a 19 años que han tenido embarazo no planificado (sobre el total de las personas de esa edad iniciadas sexualmente); vii) porcentaje de personas de 15 a 19 años que se han practicado un aborto (respecto del total de personas de esa edad iniciadas sexualmente y del total de personas de esa edad que han tenido embarazo no planificado).

Estos indicadores son tipificados por edad -usando para ello la estructura de edad simple del total de la población de 15 a 19 años- para hacer comparaciones entre grupos socioeconómicos (clasificación incluida en la Enaju basada en Esomart: ABC1, C2, C3, D y E) que controlen, al menos, la distorsión generada por las diferencias de distribución etaria simple entre estos grupos ${ }^{\mathrm{b}}$.

Para obtener una estimación de la cuantía y significación estadística de la asociación entre el grupo socioeconómico y los indicadores, se calcularon regresiones logísticas condicionando estos últimos por la edad simple y el grupo

(b) La exposición al riesgo a muchos de los eventos medidos en los indicadores varía fuertemente con la edad, por lo cual las diferencias de estructuras etarias entre grupos en comparación puede generar diferencias espurias entre los indicadores de los distintos grupos. 
socioeconómico ${ }^{\mathrm{c}}$.

Todos los tabulados y regresiones se calcularon separadamente para hombres y mujeres. Como los resultados de los primeros tienen inconsistencias -que se comentan en el análisis- el grueso del examen refiere a las mujeres. Todos los procesamientos se efectuaron con las bases de datos debidamente expandidas. La información detallada sobre tamaño muestral, tasas de respuestas y errores muestrales está disponible en los informes oficiales de las encuestas usadas, aunque estos errores, incluyendo los efectos de diseño muestral, no pudieron ser calculados en este documento por falta de antecedentes respecto del efecto diseño.

\section{RESULTADOS}

La información que proporcionan las estadísticas vitales (Figura 1) muestra un aumento de la fecundidad adolescente entre 2005 y 2008 después de, al menos, 6 años de baja. Debido a esto no resulta raro que la comparación entre la Enaju 2006 y 2009 muestre un aumento de la maternidad adolescente, ya que el indicador "porcentaje de madres" (porcentaje de mujeres de 15 a 19 años que tienen hijos), aumentó desde 8.6 a 10.7 (Figura 2).

Ahora bien, el indicador "porcentaje de

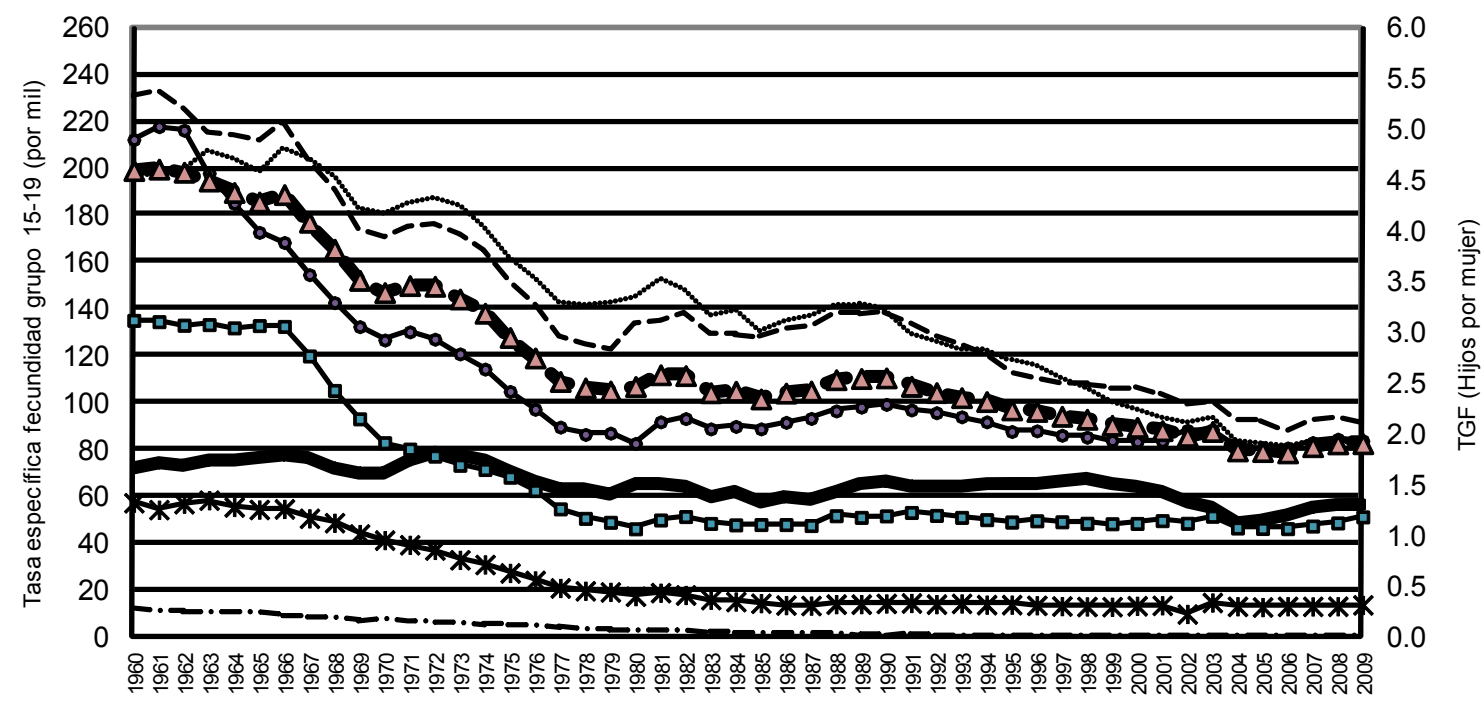

Años

$\longrightarrow$ 15-19

Fuente: cálculos del autor, basados en las estadísticas vitales del INE: Anuario Demografía hasta 1999 e Informes Anuales de Estadísticas Vitales desde 2000 a 2009.

Figura 1. Chile, 1960-2009: tasas específicas de fecundidad.

(c) Si bien las regresiones no tienen pretensiones explicativas, tanto por la ausencia de temporalidad de las variables como por la falta de otras variables importantes que lo hacen inespecífico, tiene una dirección supuesta de la relación, que es desde el nivel socioeconómico al comportamiento o subjetividad sexual y reproductiva. Los riesgos de "causalidad inversa" están presentes sobre todo en la primera relación, por cuanto la maternidad adolescente puede generar la formación de un nuevo hogar y con ello cambiar el nivel socioeconómico de los y las adolescentes. La evidencia disponible, sin embargo, sugiere que esto ocurre en la minoría de los casos, porque la situación más extendida es la permanencia de los y las adolescentes en su hogar de origen, incluso si tienen hijos. Y cuando hay formación de un nuevo hogar, no suele haber una reclasificación socioeconómica, pues tienden a recibir apoyo parental si son de familias acomodadas o mantienen condiciones de pobreza si son de familias pobres. 
madres" es afectado, como todos los indicadores sintéticos de la fecundidad adolescente (es decir del grupo de 15 a 19 años de edad), por cambios en la estructura de la población de referencia (distribución por edad simple dentro del grupo de mujeres de 15 a 19 años de edad). El cambio en la estructura etaria y socioeconómica de este grupo puede modificar el indicador sin un cambio subyacente real de la variable de interés (la probabilidad de ser madre adolescente. Para controlar este efecto se usó el procedimiento demográfico de "tipificación", consistente en aplicar el indicador por edad simple de 2009 a la estructura por edad simple de 2006, obteniendo así un nuevo porcentaje, ahora "tipificado" por edad y, por tanto, comparable. Esta comparación muestra que el aumento de la maternidad adolescente ha sido efectivo -vale decir, no es el resultado de un "efecto de composición" debido a cambios etario y socioeconómico en las muestras-, aunque algo menos pronunciado que el observado. En efecto, si la estructura etaria y socioeconómica de las mujeres de 15 a 19 años del 2009 hubiese sido la misma que hubo en 2006, el porcentaje de adolescentes con hijos habría sido de $9.9 \%$, es decir, menor que el observado (10.7\%, ver Figura 2) pero aún bastante mayor que el 8.6\% observado en 2006 (Figura 2).

Respecto de la evolución de la desigualdad de la maternidad temprana, la Figura 2 muestra un aumento relativo mayor en el grupo más acomodado (ABC1), por lo cual la desigualdad no aumenta. Con todo, sigue siendo muy elevada: el porcentaje de madres adolescentes del grupo 15-19 años más pobre (D) es 11 veces el porcentaje del grupo más acomodado $(\mathrm{ABC} 1)$; en 2006 la relación era de 12 veces.

Respecto de las desigualdades sociales de las variables intermedias y del embarazo adolescente no planificado en 2009, la Tabla 1 sintetiza la información obtenida para este trabajo. Las cifras no estandarizadas se usan y se complementan con los valores estandarizados -tipificación por edad-, los que permiten controlar el efecto distorsionador que tienen las diferencias de estructura etaria entre los grupos socioeconómicos sobre el valor de los indicadores usados en este trabajo. El análisis se focaliza en las mujeres, por cuanto sus patrones son más estilizados y consistentes en ciertas variables clave. De las Tablas 1 a 6 puede concluirse que:

a. Las adolescentes más pobres siguen teniendo una iniciación sexual más temprana que el resto de los grupos socioeco-
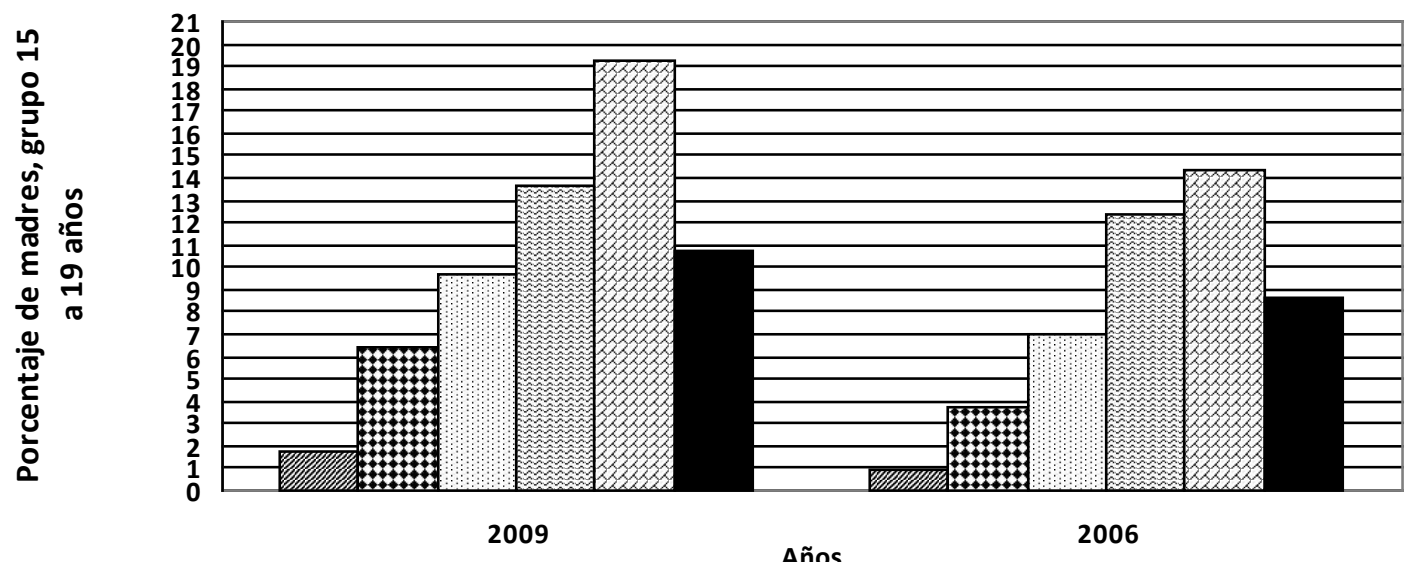

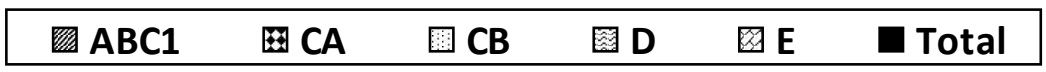

Fuente: Enaju 2006 y 2009, procesamientos especiales.

Figura 2. Chile, 2006-2009: Evolución de la maternidad adolescente total y nivel socioeconómico. 
nómicos, pero el diferencial es más bien ligero; más aún, las adolescentes del grupo más acomodado ya no se distinguen por una iniciación sexual más tardía, de hecho, el inicio de la vida sexual entre los cuatro grupos socioeconómicos distintos al más pobre, es muy similar. En el caso de los hombres, la convergencia de la iniciación sexual es incluso más acentuada. Y entre los sexos también se aprecia bastante similitud (Tabla 1). Estos datos son compatibles con las hipótesis de Bozón sobre el cambio de los comportamientos sexuales con el avance de la cultura occidental moderna ${ }^{23}$, a saber, una tendencia a la convergencia entre sexos y grupos socioeconómicos tanto en edad de iniciación sexual ( relativamente temprana), como de contexto nupcial (prematrimonial).

b. El debut sexual protegido sigue siendo muy desigual y escalonado socialmente: $77 \%$ de las adolescentes del grupo más acomodado usó anticoncepción en su primera relación sexual, lo que sólo hizo el $38 \%$ de las adolescentes del grupo más pobre; cuando se consideran los valores estandarizados, la brecha se ensancha incluso más. Entre los hombres también hay una gran desigualdad, aunque inferior a la que registran las mujeres (Tabla 2). Ciertamente, la persistencia de esta desigualdad abona la hipótesis de la "modernidad sexual truncada" usada en América Latina, que complementa las propuestas por Bozón. Esta hipótesis subraya que el abatimiento de las barreras para la sexualidad prenupcial puede adelantarse al abatimiento de las barreras de acceso a los anticonceptivos desde la primera relación sexual, con el consecuente efecto de alza de la fecundidad adolescente prenupcial.

c. La frecuencia de actividad sexual entre los iniciados/as -que se mide de manera muy gruesa en la Enaju 2009 mediante haber tenido o no al menos una relación sexual en los 12 meses previos a la encuesta- no muestra la desigualdad social del debut desprotegido. Por el contrario, los y las adolescentes más pobres son quienes tienen los porcentajes de actividad sexual menor (Tabla 3). Pese a sus limitaciones, este dato tiende a descartar que la intensidad de la vida sexual explique la desigualdad social de la maternidad adolescente; por cierto, si la menor actividad sexual de este grupo es desprotegida, entonces esta combinación sí puede explicar la desigualdad social de la maternidad adolescente.

d. En directa relación con lo expuesto en el punto previo, las cifras sobre uso de anticoncepción en la última relación sexual muestran que éste es menor entre las más pobres, siendo más marcada la desigualdad en el caso de los hombres. Ahora bien, entre los 4 grupos socioeconómicos distintos al más pobre, no aparece un patrón de desigualdad entre las mujeres, que sí se observa entre los hombres (Tabla 4). Aunque valioso, este indicador tiene al menos una debilidad clave, cual es que no controla según la condición de maternidad $\mathrm{y}$, por tanto, parte de las usuarias adolescentes ya ha tenido hijos. En estos casos, el uso en la última relación no apunta a prevenir la maternidad adolescente sino a evitar un segundo hijo durante la adolescencia.

e. El embarazo no planificado es la variable que presenta un mayor grado de desigualdad social (Tabla 5). Este hallazgo -que según nuestro conocimiento es una novedad como estimación nacional- es de la mayor relevancia conceptual y de política, ya que en ambos aspectos apoya a los enfoques que destacan las barreras de acceso y las dificultades (por inmadurez, falsas sensaciones de seguridad, conocimiento parciales o distorsionados, irresponsabilidad, impulsividad, limitaciones negociadoras, etc.) de las y los adolescentes para autoprotegerse, en desmedro de los enfoques que plantean que la causa de la mayor maternidad adolescente entre los pobres es una estrategia o un anhelo profundo. En efecto, por el tipo de pregunta usada, que no se restringe a los entrevistados que han tenido hijos sino que refiere a todas las en- 
trevistadas que se han iniciado sexualmente, el $41 \%$ de muchachas del grupo más pobre que ha experimentado, al menos, un embarazo no planificado (Tabla 5) es una cifra alarmante. Entre éstas sólo un 17\% declara haber usado anticoncepción en la primera y última relación sexual, contra un 44\% que usó en ambas ocasiones entre las muchachas iniciadas del grupo E y que no han experimentado embarazos no planificados (datos no mostrados en la Tabla). Como contrapartida, el $8.6 \%$ de embarazos no planificados entre las adolescentes del grupo más acomodado, pese a las escasas diferencias en materia de iniciación y actividad sexual con el grupo más pobre, es revelador de la mayor protección anticonceptiva de este grupo. Con todo, ni siquiera este grupo está "blindado", lo que muestra que las lagunas en materia anticonceptiva también afectan a este grupo, aunque sea de manera mucho menos intensa. En efecto, un $58 \%$ de las que experimentaron embarazo no deseado en el grupo $\mathrm{ABC} 1$ no usó anticoncepción ni en la primera ni la última relación sexual mientras que ese guarismo es sólo del 19\% para las muchachas de ese grupo que no han tenido embarazos no planificados (datos no mostrados en el cuadro).

f. Finalmente, y de nuevo como hallazgo novedoso, el aborto inducido también aparece como una variable altamente desigual, por cuanto es usado con mayor frecuencia por las mujeres de estrato alto (Tabla 6). Cabe mencionar que este resultado proviene de las mujeres que declaran haber tenido al menos un embarazo no planificado; por lo tanto, refiere a un grupo pequeño en términos relativos $(8.6 \%)$ para el estrato más acomodado $\mathrm{y}$, en cambio, atañe a un grupo mucho mayor en términos relativos $(40.7 \%)$ en el caso del grupo más pobre (Tabla 6). Por ende, el desigual uso del aborto no se traduce en una experiencia del mismo más frecuente entre las adolescentes acomodadas (de hecho, al calcular el porcentaje entre el total de iniciadas, el porcentaje baja significativamente y casi no hay diferencia entre estratos socioeconómicos) sino en un uso más común entre aquellas que parecen tener mayor urgencia para usarlo (embarazo no planificado), lo que ratifica su condición de desigualdad social.

Tabla 1. Chile, 2009, población de 15 a 19 años: porcentaje que ha tenido actividad sexual penetrativa, por nivel socioeconómico y según sexo. Valores observados y estandarizados por edad simple.

\begin{tabular}{llllll}
\hline Nivel socio-económico & \multicolumn{2}{c}{ Observado } & \multicolumn{2}{c}{ Estandarizado } \\
\hline HBC1 & Hombre & Mujer & Hombre & & Mujer \\
C2 & 46.0 & 43.0 & 47.5 & 46.4 & \\
C3 & 48.7 & 44.3 & 49.2 & 45.1 & \\
D & 50.3 & 45.5 & 51.6 & 44.5 & \\
E & 48.6 & 47.1 & 48.4 & 46.7 & \\
Total & 53.2 & 52.9 & 49.8 & 52.2 & \\
\hline
\end{tabular}

Fuente: procesamiento especial microdatos ENAJU 2009, valores expandidos 
Tabla 2. Chile, 2009, población de 15 a 19 años, sexualmente iniciada: porcentaje que usó de anticoncepción en la primera relación sexual, por nivel socioeconómico y según sexo. Valores observados y estandarizados por edad simple.

\begin{tabular}{lcccc}
\hline Nivel Socio-económico & \multicolumn{2}{c}{ Observado } & \multicolumn{2}{c}{ Estandarizado } \\
\hline & Hombre & Mujer & Hombre & Mujer \\
\hline ABC1 & 80.2 & 75.6 & 78.4 & 77.1 \\
C2 & 65.7 & 75.8 & 66.0 & 76.1 \\
C3 & 53.9 & 58.7 & 54.7 & 58.1 \\
D & 53.8 & 50.4 & 54.2 & 50.4 \\
E & 43.6 & 38.1 & 44.1 & 37.1 \\
Total & 57.9 & 58.8 & 57.9 & 58.8 \\
\hline
\end{tabular}

Fuente: procesamiento especial microdatos ENAJU 2009, valores expandidos

Tabla 3. Chile, 2009, población de 15 a 19 años, sexualmente iniciada: porcentaje que tuvo sexo en los últimos 12 meses, por nivel socioeconómico y según sexo. Valores observados y estandarizados por edad simple.

\begin{tabular}{lcccc}
\hline Nivel socio-económico & \multicolumn{2}{c}{ Observado } & \multicolumn{2}{c}{ Estandarizado } \\
\hline ABC1 & Hombre & Mujer & Hombre & Mujer \\
C2 & 95.0 & 86.2 & 86.6 & 95.6 \\
C3 & 87.0 & 88.0 & 87.8 & 86.7 \\
D & 80.2 & 85.6 & 85.1 & 80.3 \\
E & 87.9 & 89.9 & 90.0 & 87.6 \\
Total & 79.3 & 79.9 & 78.4 & 73.5 \\
\hline
\end{tabular}

Fuente: procesamiento especial microdatos ENAJU 2009, valores expandidos

Tabla 4. Chile, 2009, población de 15 a 19 años, sexualmente iniciada: porcentaje que uso anticoncepción en la última relación sexual por nivel socioeconómico y según sexo. Valores observados y estandarizados por edad simple.

\begin{tabular}{lcccc}
\hline Nivel socio- economico & \multicolumn{2}{c}{ Observado } & \multicolumn{2}{c}{ Estandarizado } \\
\hline ABC1 & Hombre & Mujer & Hombre & Mujer \\
C2 & 89.7 & 63.9 & 89.1 & 65.5 \\
C3 & 69.7 & 74.5 & 70.1 & 74.9 \\
D & 69.5 & 68.4 & 68.5 & 68.6 \\
E & 66.5 & 68.7 & 66.4 & 70.0 \\
Total & 49.4 & 59.1 & 48.7 & 57.0 \\
\hline
\end{tabular}

Fuente: procesamiento especial microdatos Enaju 2009, valores expandidos. 
Tabla 5. Chile, 2009, población de 15 a 19 años, sexualmente iniciada: porcentaje que ha tenido uno o más embarazos no planificados por nivel socioeconómico y según sexo. Valores observados y estandarizados por edad simple.

\begin{tabular}{lcccc}
\hline Nivel socio-económico & \multicolumn{2}{c}{ Observado } & \multicolumn{2}{c}{ Estandarizado } \\
\hline ABC1 & Hombre & Mujer & Hombre & Mujer \\
C2 & 5.7 & 9.7 & 6.0 & 9.8 \\
C3 & 8.6 & 17.8 & 8.7 & 18.2 \\
D & 7.0 & 19.1 & 7.3 & 18.7 \\
E & 9.3 & 34.1 & 9.1 & 33.6 \\
Total & 10.7 & 41.1 & 10.1 & 41.2 \\
\hline
\end{tabular}

Fuente: procesamiento especial microdatos Enaju 2009, valores expandidos

Tabla 6. Chile, 2009, población de 15 a 19 años, sexualmente iniciada: porcentaje que ha abortado (respecto de quienes han tenido embarazos no planificados) por nivel socioeconómico y según sexo. Valores observados y estandarizados por edad simple

\begin{tabular}{lcccc}
\hline Nivel socio-económico & \multicolumn{2}{c}{ Observado } & \multicolumn{2}{c}{ Estandarizado } \\
\hline & Hombre & Mujer & Hombre & Mujer \\
\hline ABC1 & 70.5 & 26.5 & 21.0 & 17.3 \\
C2 & 34.8 & 9.6 & 45.2 & 10.4 \\
C3 & 32.9 & 3.3 & 25.5 & 2.9 \\
D & 34.3 & 4.0 & 33.8 & 4.7 \\
E & 38.1 & 7.6 & 32.0 & 8.6 \\
Total & 36.0 & 6.2 & 36.0 & 6.2 \\
\hline
\end{tabular}

Fuente: procesamiento especial microdatos Enaju 2009, valores expandidos

Con el propósito de examinar la forma y la significación estadística $(\mathrm{p}<0.01)$ del efecto del nivel socioeconómico sobre las variables condicionadas usadas en este estudio, se presentan los coeficientes (odd ratios) de sendas regresiones logísticas bionomiales para mujeres de 15 a 19 años de edad que controlan por la edad simple dentro de este grupo. La muestra no expandida se usa para estos cálculos, para evitar significaciones estadísticas artificiales. El grupo más pobre (Nivel socioeconómico E) se usa como categoría de referencia. Los resultados ratifican las conclusiones elaboradas con base en la Tabla 1. Hay asociación estadística significativa entre el nivel socioeconómico y las chances de tener un hijo en este grupo de edad: las chances del grupo $\mathrm{ABC} 1$ son sólo un $8 \%$ de las del grupo E (Tabla 7). No hay diferencias estadísticamente significativas entre grupos socioeconómicos en materia de iniciación sexual (Tabla 8). Hay asociación estadística significativa entre el nivel socioeconómico y las chances de tener un debut sexual protegido: las chances del grupo $\mathrm{ABC} 1$ son 5 veces las del grupo E (Tabla 9). Hay diferencias estadísticamente significativas entre grupos socioeconómicos en materia de embarazo no planificado: el grupo $\mathrm{ABC} 1$ tiene un sexto de las chances del grupo E (Tabla 10). Pese a la magnitud del coeficiente en el caso del uso de aborto (el grupo ABC1 tiene 4.7 veces la chance del grupo E), no hay diferencias estadísticamente significativas ${ }^{\mathrm{d}}$ en-

(d) Esto se debe a que el grupos $\mathrm{ABC} 1$ tiene muy pocos casos, ya que la población de referencia tiene dos filtros: a) ser sexualmente iniciada ( $47.3 \%$ de este grupo) y b) haber tenido embarazos no deseados $(8.6 \%$ del $47.3 \%$ de iniciadas). 
tre grupos socioeconómicos al respecto (Tabla 11).

Tabla 7. Chile, 2009: Regresión logística binomial de la edad y el nivel socioeconómico sobre la condición de maternidad (haber tenido hijos), mujeres de 15 a 19 años.

\begin{tabular}{lcccccc}
\hline Variables & B & Error estándar & Test de Wald & $\begin{array}{c}\text { Grados de } \\
\text { libertad }\end{array}$ & $\begin{array}{c}\text { Signi-fica- } \\
\text { ción. }\end{array}$ & $\begin{array}{c}\text { (B) Expo- } \\
\text { nencial (odds } \\
\text { ratios) }\end{array}$ \\
\hline EDAD & 0.64273 & 0.003318 & 37534.53 & 1 & .000 & $\mathbf{1 . 9 0 1 6 6 5}$ \\
E & & & 14606.32 & 4 & & \\
ABC1 & -2.54531 & 0.037041 & 4721.845 & 1 & .003 & $\mathbf{0 . 0 7 8 4 4 9}$ \\
C2 & -1.27733 & 0.012992 & 9666.45 & 1 & .000 & $\mathbf{0 . 2 7 8 7 8 1}$ \\
C3 & -0.87968 & 0.013358 & 4336.678 & 1 & .005 & $\mathbf{0 . 4 1 4 9 1 5}$ \\
D & -0.39895 & 0.011047 & 1304.242 & 1 & .125 & 0.671022 \\
Constante & -12.6162 & 0.059685 & 44681.92 & 1 & .000 & $3.32 \mathrm{E}-06$ \\
\hline
\end{tabular}

Fuente: procesamiento especial microdatos Enaju 2009.

Nota: en negrita coeficientes estadísticamente significativos con $p<0.01$. Cálculos efectuados con la muestra no expandida, 1864 casos válidos

Tabla 8. Chile, 2009: Regresión logística binomial de la edad y el nivel socioeconómico sobre la condición de actividad sexual (haber tenido), mujeres de 15 a 19 años.

\begin{tabular}{lcccccc}
\hline Variables & B & Error estándar & Test de Wald & $\begin{array}{c}\text { Grados de } \\
\text { libertad }\end{array}$ & $\begin{array}{c}\text { Signi-fica- } \\
\text { ción }\end{array}$ & $\begin{array}{c}\text { (B) Expo- } \\
\text { nencial (odds } \\
\text { ratios) }\end{array}$ \\
\hline EDAD & 0.579135 & 0.001907 & 92188.8 & 1 & .000 & $\mathbf{1 . 7 8 4 4 9 3}$ \\
E & & & & 4 & & \\
ABC1 & -0.25937 & 0.01268 & 418.392 & 1 & .385 & 0.771538 \\
C2 & -0.31448 & 0.008851 & 1262.394 & 1 & .131 & 0.730165 \\
C3 & -0.35017 & 0.009577 & 1336.792 & 1 & .120 & 0.704566 \\
D & -0.22897 & 0.008626 & 704.5963 & 1 & .259 & 0.795355 \\
Constante & -9.70836 & 0.033159 & 85720.74 & 1 & .000 & $6.08 \mathrm{E}-05$ \\
\hline
\end{tabular}

Fuente: procesamiento especial microdatos Enaju 2009.

Nota: en negrita coeficientes estadísticamente significativos con $p<0.01$. Cálculos efectuados con la muestra no expandida, 1854 casos válidos 
Tabla 9. Chile, 2009: Regresión logística binomial de la edad y el nivel socioeconómico sobre la condición de uso de anticoncepción en la primera relación sexual (haber usado anticoncepción), mujeres de 15 a 19 años iniciadas sexualmente.

\begin{tabular}{lcccccc}
\hline Variables & B & Error estándar & Test de Wald & $\begin{array}{c}\text { Grados de } \\
\text { libertad }\end{array}$ & Signi-ficación & $\begin{array}{c}\text { (B) Exponencial } \\
\text { (odds ratios) }\end{array}$ \\
\hline EDAD & 0.081658 & 0.002814 & 842.2918 & 1 & .217 & 1.085084 \\
E & & & & & &. \\
ABC1 & 1.644967 & 0.019281 & 7278.376 & 1 & .000 & $\mathbf{5 . 1 8 0 8 4 1}$ \\
C2 & 1.638738 & 0.012386 & 17504.31 & 1 & .000 & $\mathbf{5 . 1 4 8 6 6 7}$ \\
C3 & 0.919717 & 0.012943 & 5049.364 & 1 & .003 & $\mathbf{2 . 5 0 8 5 8}$ \\
D & 0.524784 & 0.011361 & 2133.747 & 1 & .050 & 1.690094 \\
Constante & -1.92116 & 0.050466 & 1449.231 & 1 & .106 & 0.146436 \\
\hline
\end{tabular}

Fuente: procesamiento especial microdatos Enaju 2009.

Nota: en negrita coeficientes estadísticamente significativos con $\mathrm{p}<0.01$. Cálculos efectuados con la muestra no expandida, 831 casos válidos

Tabla 10. Chile, 2009: Regresión logística binomial de la edad y el nivel socioeconómico sobre la experiencia de embarazo no deseado (haber tenido embarazo no planificado), mujeres de 15 a 19 años que han tenido al menos un embarazo.

\begin{tabular}{lcccccc}
\hline Variables & B & $\begin{array}{c}\text { Error Están- } \\
\text { dar }\end{array}$ & Test de Wald & $\begin{array}{c}\text { Grados } \\
\text { de Liber- } \\
\text { tad }\end{array}$ & Significación & $\begin{array}{c}\text { (B) Expo- } \\
\text { nencial (odds } \\
\text { ratios) }\end{array}$ \\
\hline EDAD & 0.240817 & 0.003299 & 5329.446 & 1 & .002 & $\mathbf{1 . 2 7 2 2 8 9}$ \\
E & & & & & & \\
ABC1 & -1.87896 & 0.026097 & 5183.788 & 1 & .002 & $\mathbf{0 . 1 5 2 7 4 9}$ \\
C2 & -1.19791 & 0.013027 & 8456.323 & 1 & .000 & $\mathbf{0 . 3 0 1 8 2 5}$ \\
C3 & -1.14184 & 0.014372 & 6312.303 & 1 & .001 & $\mathbf{0 . 3 1 9 2 3 1}$ \\
D & -0.32247 & 0.011581 & 775.2834 & 1 & .237 & 0.724361 \\
Constante & -4.55004 & 0.058889 & 5969.848 & 1 & .001 & 0.010567 \\
\hline
\end{tabular}

Fuente: procesamiento especial microdatos Enaju 2009.

Nota: en negrita coeficientes estadísticamente significativos con $\mathrm{p}<0.01$. Cálculos efectuados con la muestra no expandida, 822 casos válidos

\section{DISCUSIÓN}

Este trabajo se inscribe en un esfuerzo sistemático de varios años tendientes a dilucidar los factores que condicionan la aún alta frecuencia de la maternidad adolescente y su marcada desigualdad social. La evidencia presentada en esta ocasión refuerza la línea argumental de que el factor decisivo son las barreras de acceso a anticoncepción desde la primera relación sexual combinadas con lagunas y debilidades para su uso sistemático. Como ambos factores se distribuyen muy desigualmente entre los grupos socioeconómicos, el resultado es que la desigualdad también se expresa en embarazos no planificados, lo que fundamenta desde el punto de vista del ejercicio de derechos reproductivos de los y las adolescentes una acción pública decidida en la materia.

Las políticas públicas pueden ser claves tanto para abatir barreras de acceso como para aumentar las capacidades preventivas de los 
Desigualdades y convergencias sociales en materia sexual y reproductiva entre adolescentes - Jorge Rodríguez et al

Tabla 11. Chile, 2009: Regresión logística binomial de la edad y el nivel socioeconómico sobre la experiencia de aborto (haber abortado), mujeres de 15 a 19 años que han tenido al menos un embarazo no planificado.

\begin{tabular}{lcccccc}
\hline Variables & B & Error estándar & Test de Wald & $\begin{array}{c}\text { Grados de } \\
\text { libertad }\end{array}$ & Significación & $\begin{array}{c}\text { (B) Exponencial } \\
\text { (odds ratios) }\end{array}$ \\
\hline EDAD & 0.10433 & 0.01259 & 68.6577 & 1 & .725 & 1.10996 \\
E & & & & & & \\
ABC1 & 1.54547 & 0.05952 & 674.205 & 1 & .270 & 4.69017 \\
C2 & 0.2081 & 0.03863 & 29.0166 & 1 & .819 & 1.23133 \\
C3 & -0.9829 & 0.06037 & 265.105 & 1 & .489 & 0.37423 \\
D & -0.7204 & 0.03818 & 356.036 & 1 & .422 & 0.48657 \\
Constante & -4.3131 & 0.22197 & 377.556 & 1 & .409 & 0.01339 \\
\hline
\end{tabular}

Fuente: procesamiento especial microdatos Enaju 2009.

Nota: En esta regresión ningún coeficiente es significativo con p-value $<0.05$

y las adolescentes. Programas de educación y salud sexual y reproductiva activos y sensibles a las especificidades de los y las adolescentes son las herramientas genéricas para ello. Sin embargo, su éxito está mediado por otros factores como las dinámicas familiares, los mensajes de los medios de comunicación, las modas, tribus y subculturas juveniles, las aproximaciones de los adultos a la sexualidad de lo/as adolescentes, las complejidades de la adolescencia incluyendo las relaciones de pareja y las mismas faltas de oportunidades que experimentan los y las adolescentes, en particular lo/as más pobres. Esta complejidad se revela en toda su magnitud cuando se examinan las respuestas que los y las adolescentes dan por no haber usado anticonceptivos en su última relación sexual: lejos de ser porque querían tener hijos (sólo 3\%) es la irresponsabilidad la causa más nombrada - un asunto en que cabe profundizar en futuras investigaciones- seguida de otras que tienen que ver barreras de acceso y prejuicios hacia el uso de anticonceptivos. Hay, por tanto, una tarea más compleja a largo plazo, pero esta en modo alguno debe conducir al fatalismo o a la inacción en el corto plazo.

\section{AGRADECIMIENTOS}

Los autores agradecen a Gabriela Marise de Oliveira Bonifacio, Magister en Demogra- fía de Cedeplar, Universidad Estadual de Minas Gerais, Brasil, por el apoyo brindado para el procesamiento de datos (gabriela@cedeplar.ufmg.br). Asimismo, agradecen a Javiera Rauld, estudiante de Sociología de la Universidad de Chile, que brindó apoyo a la revisión del texto durante su práctica profesional, realizada en Celade bajo la supervisión de Jorge Rodríguez. Finalmente, también agradecen al Instituto Nacional de la Juventud de Chile, que entregó la base de microdatos de la encuesta en formato SPSS luego de una solicitud oficial por parte de los autores.

\section{REFERENCIAS}

1. RODRÍGUEZ J. Reproducción adolescente $y$ desigualdades en Chile: tendencias, determinantes y opciones de política. Revista de Sociología de la Universidad de Chile 2010, 23:39-65.

2. MOLINA R, MOLINA T, y GONZÁLEZ E. Salud sexual y reproductiva en la adolescencia. Santiago: Mediterráneo; 2003.

3. RODRÍGUEZ J. Reproducción en la adolescencia: el caso de Chile y sus implicaciones de política. Revista de la CEPAL. 2005; 86:123-146.

4. DIDES C, BENAVENTE C, MORAN JM. Diagnóstico de la situación del embarazo en la adolescencia en Chile. Edición 11. Santiago: FLACSO-Chile/ Ministerio de Salud/UNFPA 2009; 2008.

5. HOFFMEISTER L. Diagnóstico Situación de Salud de Adolescentes y Jóvenes en Chile 2007. Santiago: Ministerio de Salud de Chile; 2007. 
6. MOLINA R, LUENGO X, SANDOVAL J, GONZALEZ E, MOLINA. T Factores de Riesgo del embarazo, parto y recién nacido en adolescentes embarazadas. Rev. Chil Obst Ginecol Inf y Adolec. 1998; 5: 17-28.

7. MOLINA M, FERRADA C, PÉREZ R, CID L, CASANUEVA V, GARCÍA A. Rev. méd. Chile [online]. 2004, vol.132, n.1 [citado 201105-08], pp. 65-70. Disponible en: <http:// www.scielo.cl/scielo.php?script=sci_arttext\& pid $=$ S0034-98872004000100010\&lng $=$ es\&nrm $=$ iso>. ISSN 0034-9887. doi: 10.4067/S003498872004000100010 .

8. SIDEBOTTOM A, BIRNBAUM A, STODDARD S. Decreasing Barriers for Teens: Evaluation of a New Teenage Pregnancy Prevention Strategy in SchoolBased Clinics. American Journal of Public Health. 2003 November; 93(11): 180-183.

9. RODRÍGUEZ J, HOPENHAYN M. Maternidad adolescente en América Latina y el Caribe. Tendencias, problemas y desafíos. Santiago: CEPAL, Boletín Desafíos. 2007; 4: 4-9.

10. MOLINA R, MOLINA G, GONZÁLEZ E. Madres niñas-adolescentes de 14 años y menos. Un grave problema de salud pública no resuelto en Chile. Revista Médica Chilena. 2007; 135: 79-86.

11. JEEJEBOY S, BOTT S. Non-Consensual Sexual Experiences of Young People: A Review of the Evidence from Developing Countries. New Delhi: Population Council; 2003. Disponible en: www.popcouncil.org/pdfs/wp/seasia /seawp16.pdf

12. LUENGO X, GONZÁLEZ E. Hijos de madres adolescentes. En: MOLINA R, SANDOVAL J, GONZÁLEZ E. 2003. op. Cit.

13. LUENGO X. Características de la adolescencia normal. En: MOLINA R, SANDOVAL J, GONZÁLEZ E. 2003. op. Cit.

14. RODRÍGUEZ J, JIMENEZ M, ALIAGA L. Una mirada desde América Latina y el Caribe al Objetivo de Desarrollo del Milenio de acceso universal a la salud reproductiva. Santiago: CELADE Serie Población y Desarrollo 97; 2011.

15. DI CESARE M, RODRÍGUEZ J. Análisis micro de los determinantes de la fecundidad adolescente en Brasil y Colombia. Universidad Autónoma del Estado de México: Papeles de Población. 2006; 48:107-140.

16. BREIBAUER C, MADDALENO M. Youth: choices and change. Promoting healthy behaviors in adolescents. Washington: PAHO; 2005.

17. DULANTO E. El adolescente. México: McGrawHill Interamericana; 2000.

18. BONGAARTS J, COHEN B. Adolescent reproductive behavior in the developing World. Studies in Family Planning 1998, 29(2): 99-105.

19. RENDALL M, ARCACIL E, BAGAVOS C, COUET C, DEROSE A, ET AL. Increasingly heterogeneous ages at first birth by education in Southern European and Anglo-American family-policy regimes: A seven-country comparison by birth cohort. Population Studies. 2010; 64 (3): 209-227.

20. FLÓREZ C, NÚÑEZ J. Teenage childbearing in Latin American countries. En DURYEA S, COX A, URETA M. Critical decision at a critical age. Adolescents and young adults in Latin America. Washington. BID; 2003.

21. FURSTENBERG F. When Will Teenage Childbearing Become a Problem? The Implications of Western Experience for Developing Countries. Studies in Family Planning Population Council. 1998; 29 (2): 246-253.

22. RODRÍGUEZ J. Reproducción adolescente $\mathrm{y}$ desigualdades en América Latina y el Caribe: un llamado a la reflexión y a la acción, Madrid: OIJCEPAL-UNFPA; 2009

23. BOZON M. A quel âge les femmes et les hommes commencent-ils leur vie sexuelle? Comparaisons mondiales et évolutions récentes. Population et sociétés. 2003; 391: 1-4.

Usted puede comentar éste y otros artículos publicados en la Revista Chilena de Salud Pública, enviando un correo electrónico a revistasp@med.uchile.cl 